\title{
Gas Flow in Microchannels - A Lattice Boltzmann Method Approach
}

\author{
Y.H. Zhang, ${ }^{1}$ R.S. Qin, ${ }^{1}$ Y.H. Sun, ${ }^{1}$ R.W. Barber, ${ }^{1}$ and D.R. Emerson ${ }^{1}$
}

Received October 12, 2004; accepted July 26, 2005

\begin{abstract}
Gas flow in microchannels can often encounter tangential slip motion at the solid surface even under creeping flow conditions. To simulate low speed gas flows with Knudsen numbers extending into the transition regime, alternative methods to both the Navier-Stokes and direct simulation Monte Carlo approaches are needed that balance computational efficiency and simulation accuracy. The lattice Boltzmann method offers an approach that is particularly suitable for mesoscopic simulation where details of the molecular motion are not required. In this paper, the lattice Boltzmann method has been applied to gas flows with finite Knudsen number and the tangential momentum accommodation coefficient has been implemented to describe the gas-surface interactions. For fully-developed channel flows, the results of the present method are in excellent agreement with the analytical slip-flow solution of the NavierStokes equations, which are valid for Knudsen numbers less than 0.1. The present paper demonstrates that the lattice Boltzmann approach is a promising alternative simulation tool for the design of microfluidic devices.
\end{abstract}

KEY WORDS: Lattice Boltzmann equation; accommodation coefficient; Knudsen number; rarefied gas dynamics; microfluidics.

\section{INTRODUCTION}

Miniaturization has revolutionized many scientific areas and associated disciplines. Micron-sized systems, commonly referred to as Micro-ElectroMechanical Systems (MEMS) or Micro-Total Analysis Systems ( $\mu$ TAS), are of particular importance in chemical, biological and clinical analyses. Not only can miniaturization significantly increase yields but it can also reduce process time and reagent consumption. Microsystems can also

\footnotetext{
${ }^{1}$ Centre for Microfluidics and Microsystems Modelling, CCLRC Daresbury Laboratory, Warrington, WA4 4AD, UK; e-mail: y.zhang@dl.ac.uk
} 
introduce enhanced functionality and enable new paradigms for highthroughput analyses.

Some surprising and curious physical effects occur in microflows that do not happen, or are not important, under more conventional circumstances. ${ }^{(1)}$ For gas flows in microsystems, the continuum hypothesis, which underpins the Navier-Stokes equations, may be inappropriate. This is because the mean free path of the gas molecules may be comparable to the length scale of the device. The Knudsen number, $K n$, which is the ratio of the mean free path of the gas molecules to the characteristic length scale of the device, is a convenient measure of the degree of rarefaction of the flow. Free-molecular flow starts when $K n>10$ whereas Navier-Stokes models are generally valid if $K n<0.01$, but can be extended into the slip-flow regime $(0.01<K n<0.1)$ by appropriate treatment of the wall boundary. The transition-flow regime lies between the slip-flow and freemolecular regimes $(0.1<K n<10)$. In practice, gas flows in long microchannels may encounter a wide range of conditions that include the continuum, slip and transition regimes. The direct simulation Monte Carlo (DSMC) method could be used for low Knudsen number flows but will be computationally expensive for low-speed problems. Hybrid algorithms, that efficiently couple DSMC and Navier-Stokes methods, offer the potential to model these mixed flow regimes. ${ }^{(2)}$ However, large errors can arise from inappropriate assumptions regarding, for example, the velocity distribution of the gas molecules at the matching interface between the two solutions. ${ }^{(3)}$ In addition, hybrid methods entail intensive computational effort for three-dimensional flow simulations.

For practical microsystem design, computational efficiency and simulation accuracy has to be balanced. Since Navier-Stokes solvers have been extensively developed and are widely available, significant effort has been made to improve slip models in order to extend the validity of the NavierStokes equations beyond $K n=0.1$. Another possible continuum approach is based on Burnett-type equations which involve terms of second-order, or higher, in Knudsen number. The difficulty is how to construct complicated constitutive laws for higher-order correlations. Our recent work comparing various analytical models for the drag force acting on a microsphere has revealed that simulations using the Navier-Stokes equations with a slip boundary condition start to differ significantly against experimental measurements at Knudsen numbers as low as 0.05 , while kinetic models produce accurate predictions. ${ }^{(4)}$ In engineering applications, macroscopic flow quantities such as shear stresses, wall slip-velocities, and mass flow rates are the most important parameters to be determined. Consequently, molecular simulations such as Molecular Dynamics (MD), DSMC, or direct numerical simulation of the Boltzmann equation are often considered too expensive 
in terms of computational cost for most practical engineering applications where the microscopic details are not required.

Recently, a lattice Boltzmann equation (LBE) approach has been used to simulate gas flows in microchannels. ${ }^{(5-7)} \mathrm{He}$ and $\mathrm{Luo}^{(8)}$ and $\mathrm{Abe}^{(9)}$ showed that the lattice Boltzmann approach can be derived from the continuous Boltzmann equation. The LBE technique on a regular lattice is a subset of the discrete velocity method used to solve the Boltzmann equation with the Bhatnagar-Gross-Krook (BGK) collision operator. It has been shown that the LBE approach is equivalent to solving a discrete Boltzmann equation with a specific finite-difference scheme. ${ }^{(10)}$ The intrinsic kinetic nature of the LBE method makes it an ideal choice for microflows where both microscopic and macroscopic behavior are important. Although bulk gas velocities in microsystems are usually very low, so that the flows are almost incompressible, the Knudsen number often spans a wide range and can easily enter the transition regime. LBE techniques could offer significant advantages over other high $K n$ solution methods because its efficiency is comparable to a Navier-Stokes solver yet it has the potential of providing accurate results beyond the slip-flow regime. The LBE approach has been proven to recover the Navier-Stokes and Burnetttype equations $^{(11)}$ and may therefore be a suitable alternative for gas flows in microdevices where slip motion at the wall surface is important.

Lattice Boltzmann methods have been used extensively to simulate incompressible fluid flows with no-slip boundary conditions but little work has been carried out on the simulation of gas flows through microchannels. Our previous work has briefly discussed the implementation of gas-slip motion at the solid surface and the definition of the Knudsen number in terms of LBE parameters. ${ }^{(12)}$ The simulation results, based on a diffusive reflection model, accurately described flow in the slip regime and qualitatively captured the Knudsen minimum phenomenon in the transition regime. The present work discusses the tangential momentum accommodation coefficient (TMAC) and its implementation into a LBE model. In addition, the correlation between the nondimensional slip velocity, Knudsen number, and TMAC are numerically tested and compared to first- and second-order analytical solutions of the Navier-Stokes equations.

\section{THEORY}

Among the LBE methods reported in the literature, the most widely used is the BGK model which utilises a single relaxation time approximation. For the sake of simplicity, the lattice BGK model is given as an example:(13) 


$$
f_{i}\left(x+c_{i} \delta_{t}, t+\delta_{t}\right)-f_{i}(x, t)=-\frac{1}{\tau}\left[f_{i}(x, t)-f_{i}^{\mathrm{eq}}(x, t)\right], \quad i=0,1, \ldots n,
$$

where $f_{i}(x, t)$ is the density distribution function along the $i$ direction at lattice site $x$ and time $t, \delta_{t}$ the time step, $c_{i}$ the discrete velocity, $\tau$ the dimensionless LBE relaxation time given by $\lambda / \delta_{t}$ where $\lambda$ is the relaxation time, and $f_{i}^{\text {eq }}$ is the local Maxwellian distribution function expressed as a Taylor expansion to second-order in fluid velocity. The density $\rho$ and bulk velocity $u$ can be determined from $\sum_{i=0}^{n} f_{i}$ and $\sum_{i=0}^{n} c_{i} f_{i} / \rho$, respectively. The LBE relaxation time $\tau$ can be related to the kinematic viscosity $v$ by $c_{s}^{2} \delta_{t}(\tau-0.5)$ for a square lattice, where $c_{s}$ is the so-called sound speed of the lattice fluid. The term -0.5 is a correction to make the LBE technique a second-order method for solving incompressible flows. ${ }^{(14)}$ In the popular nine-velocity square lattice model (D2Q9), the particle velocities, $c_{i}$, are given by Qian et al.:(13)

$$
\begin{aligned}
& c_{0}=0, \\
& c_{i}=c[\cos ((i-1) \pi / 2), \sin ((i-1) \pi / 2)], \quad i=1,2,3,4, \\
& c_{i}=\sqrt{2} c[\cos ((i-5) \pi / 2+\pi / 4), \sin ((i-5) \pi / 2+\pi / 4)], \quad i=5,6,7,8,
\end{aligned}
$$

where $c=\delta_{x} / \delta_{t}$. The equilibrium distribution for the D2Q9 model is given by Qian et al.:(13)

$$
\begin{aligned}
& f_{i}^{\mathrm{eq}}=\rho \omega_{i}\left[1+\frac{3}{c^{2}} c_{i} \cdot \mathbf{u}+\frac{9}{2 c^{4}}\left(c_{i} \cdot \mathbf{u}\right)^{2}-\frac{3}{2 c^{2}} \mathbf{u} \cdot \mathbf{u}\right], \quad \omega_{0}=\frac{4}{9}, \quad \omega_{i}=\frac{1}{9}, \\
& i=1,2,3,4, \quad \omega_{i}=\frac{1}{36}, \quad i=5,6,7,8
\end{aligned}
$$

The mean velocity of the gas molecules can be given by $\bar{c}=\sqrt{8 k T / \pi m}$, ${ }^{(15)}$ where $k$ is the Boltzmann constant, $m$ the molecular mass, and $T$ is the temperature. The mean free path, $l$, is equal to the mean distance a molecule travels between consecutive collisions, i.e. $l=\bar{c} \lambda .{ }^{(16)}$ For uniform lattice spacing, the channel height, $H$, is given by $H=N_{H} \delta_{x}$, where $N_{H}$ is the number of lattice sites and $\delta_{x}$ is the lattice spacing. Since $\tau=\lambda / \delta_{t}$, and $c=\delta_{x} / \delta_{t}=\sqrt{3 R T}$ for a D2Q9 model, where $R$ is the universal gas constant, it can be shown that

$$
K n=\frac{l}{H}=\frac{\bar{c} \lambda}{H}=\sqrt{\frac{8}{3 \pi}} \frac{\tau}{N_{H}} .
$$


Introducing the correction factor of -0.5 from the discretization of the Boltzmann equation, ${ }^{(14)}$ leads to

$$
K n=\sqrt{\frac{8}{3 \pi}} \frac{(\tau-0.5)}{N_{H}} .
$$

Equation (5) is valid for uniform lattice models such as D2Q9 or D3Q27. It should be noted that the collision interval takes no account of the persistence of velocities after collisions in the BGK model, ${ }^{(16)}$ so that the mean free path has to be defined using macroscopic properties for comparison with other results. In previous work, ${ }^{(12)} \mathrm{Eq}$. (5) was derived using an approach that related the mean free path to the dynamic viscosity, $\mu$, and macroscopic pressure, $p$, i.e. $l=(\mu / p) \sqrt{\pi k T / 2 m}$, and then considered scale effects due to the use of properties based on this definition of the mean free path. ${ }^{(15)}$ The present model removes the need for a free parameter to tune the simulation results. In order to solve the Boltzmann equation, the distribution function of the gas molecules leaving the wall surface, $f^{+}$, needs to be related to the incident molecular distribution function, $f^{-}$, which can be generally expressed by a scattering kernel, $R\left(r, v^{i} \rightarrow v\right)$, where $r$ is the position vector, $v^{i}$ is the incident velocity, and $v$ is the reflected velocity. Further details are given by $\operatorname{Cercignani}^{(17)}$ and Cercignani et al. ${ }^{(18)}$ The most widely applied kernel is the diffusive scattering model, ${ }^{(15)}$ which is given by

$$
R\left(v^{i} \rightarrow v\right)=\frac{m^{2} v_{n}}{2 \pi\left(k T_{w}\right)^{2}} \exp \left(-\frac{m v^{2}}{2 k T_{w}}\right)
$$

where $T_{w}$ is the surface temperature, and $v_{n}$ is the normal component of the incident velocity. This can be interpreted from the perspective of the gas molecules losing all information about their state before their collisions; they are therefore reflected in a state obeying the Maxwellian distribution function. Maxwell ${ }^{(19)}$ expanded this diffusive kernel to a partly diffusive $\alpha$ and partly specular $(1-\alpha)$ kernel, which has been extensively applied and tested for solving the Boltzmann equation.

To implement a gas-surface interaction model within the lattice Boltzmann method, it is not necessary to know the scattering kernel. The degree of freedom in the momentum space is very limited in the LBE approach; for example, a D2Q9 model only has nine discrete velocities. The gas-solid wall collisions need to be approximated by a combination of these velocities. Therefore, a coefficient representing the average gas-surface interaction may be sufficient. For gas-phase microfluidic flow, 
the TMAC is the most important accommodation coefficient, and can be interpreted as: ${ }^{(20)}$

$$
\sigma=\frac{M_{i}-M_{r}}{M_{i}-M_{w}}
$$

where $M$ is the tangential momentum of the molecules and the subscripts $i, r, w$ refer to the incident, reflected and wall molecules, respectively. If the wall is stationary, $M_{w}$ is zero. The TMAC, $\sigma$, will be unity for diffuse reflection and zero for specular reflection. It can be shown that $\sigma$ is the same as $\alpha$ in the Maxwellian kernel. ${ }^{(15)}$ With information for the TMAC available in the literature, a gas-surface interaction model for the LBE method can be established with some confidence. Recent work has revealed that the reflections are not always fully diffuse and the accommodation coefficient $\sigma<1.0 .^{(20)}$ In the present paper, a Maxwellian approach has been used to describe the collision characteristics in the LBE model. A representative particle collides with the wall as shown in Fig. 1. The post collision direction usually lies between the normal direction, $n$, and the specular reflection direction, $s$. On a uniform lattice with spacing $\delta$, the TMAC boundary condition at the upper wall can be implemented in a LBE (D2Q9) model as follows

$$
\begin{aligned}
& f_{8}\left(x, y, t+\delta_{t}\right)=(1-\sigma) f_{5}(x-\delta, y, t), \\
& f_{7}\left(x, y, t+\delta_{t}\right)=(1-\sigma) f_{6}(x+\delta, y, t) \\
& f_{4}\left(x, y, t+\delta_{t}\right)=\sigma f_{5}(x-\delta, y, t)+\sigma f_{6}(x+\delta, y, t)+f_{2}(x, y, t) .
\end{aligned}
$$

The lower wall boundary condition can be derived in an analogous manner. For diffuse reflection, it can be seen from Eq. (8) that the average tangential momentum is zero, as expected. The implementation of diffuse

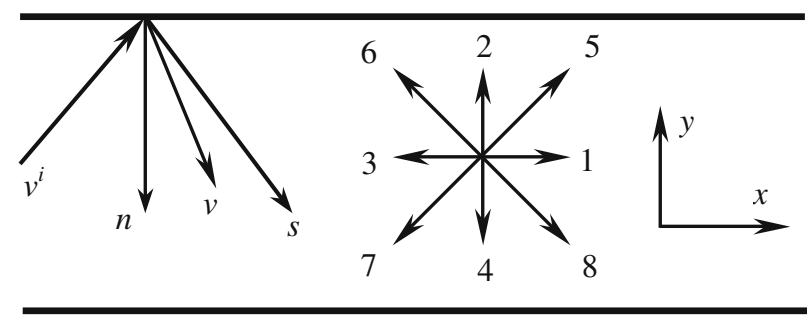

Fig. 1. Schematic diagram of gas-surface interaction and velocity directions of a twodimensional (D2Q9) model, where $v^{i}$ is the incident velocity and $v$ is the reflected velocity of a molecule. Here, $n$ is the normal direction and $s$ refers to the specular reflection direction. 
kinetic boundary conditions can be realised by discretizing the discrete velocity set, as reported in refs. 7 and 21 . In the LBE method, the degree of freedom of the velocity vectors is very limited and a fictitious particle represents a large number of gas molecules. Therefore, at the mesoscopic level, molecules reflected diffusely will exhibit an ensemble behavior such that the average tangential momentum is zero. The boundary condition given by Eq. (8) is proposed in a spirit similar to the combination of the bounce back rule and specular reflection reported by Succi. ${ }^{(22)}$ Previous studies of slip flow in gas microsystems have investigated bounce-back ${ }^{(5)}$ and specular bounce-back ${ }^{(6)}$ boundary conditions but did not take into account the effect of the accommodation coefficient. The boundary condition given by Eq. (8) can be generalized to various geometric conditions and lattice models.

\section{RESULTS AND DISCUSSION}

In this section, the numerical results of the LBE model (D2Q9) are presented for two-dimensional fully-developed channel flow. The effect of $K n$ and TMAC on the flow can be clearly distinguished in Fig. 2. For the same $K n$, decreasing the TMAC leads to increased wall-slip but a lower maximum velocity at the center of the channel. Conversely, if the TMAC is held constant, increasing the Knudsen number will lead to increased wall-slip as expected. Figure 3 illustrates that the simulation results are essentially independent of the lattice number. In Fig. 3a, the Knudsen number is 0.05 and the TMAC is 1.0 while in Fig. 3b, these values are 0.025 and 0.7 , respectively. The slip velocities in the two figures are similar
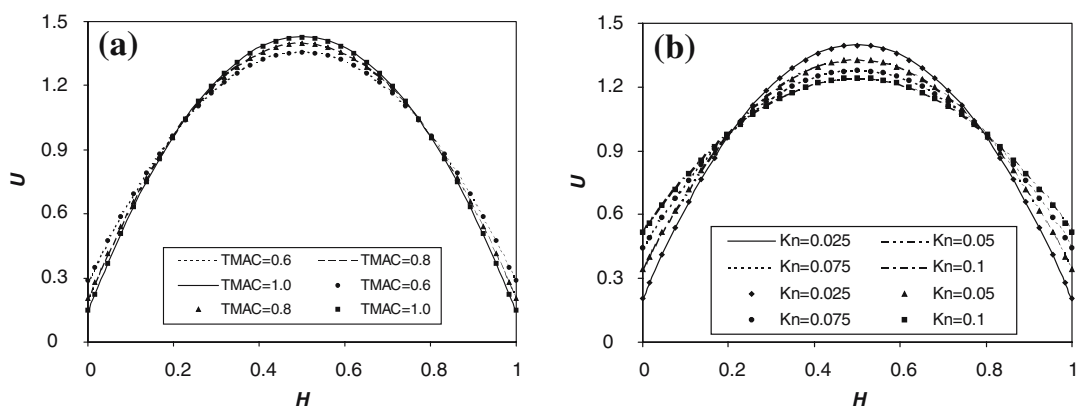

Fig. 2. The effect of TMAC and $K n$ on the velocity profile (a) $K n=0.025$ and (b) TMAC $=0.8$. Solid symbols represent the analytical solution of the Navier-Stokes equation with Cercignani's second-order slip boundary condition ${ }^{(23)}$ while lines represent the present LBE results. 

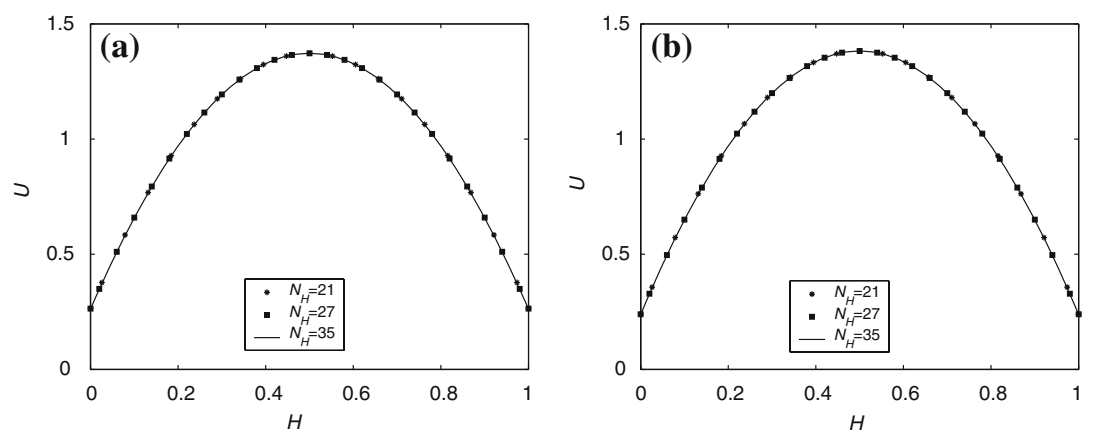

Fig. 3. The effect of the lattice number, $N_{H}$, across the channel height. Fully-developed channel flow with (a) $K n=0.05, \sigma=1.0$ and (b) $K n=0.025, \sigma=0.7$.

despite the fact that the Knudsen numbers are different. Therefore, for fully-developed gas flows in a microchannel, the velocity profiles clearly depend upon an interaction between the Knudsen number and the TMAC. This observation is consistent with both theoretical results and experimental data available in the literature. ${ }^{(20)}$

In the slip-flow regime $(K n<0.1)$, the Navier-Stokes equations, with first- or second-order boundary conditions, are considered acceptable. Cercignani $^{(23)}$ used a BGK approximation and obtained a second-order slip model for rarefied gas flows. Subsequently, Hadjiconstantinou ${ }^{(24)}$ rescaled and improved the model for a hard sphere gas by considering Knudsen layer effects and obtained

$$
u_{w}=\left.1.1466 l \frac{\partial u}{\partial n}\right|_{\text {wall }}-\left.0.31 l^{2} \frac{\partial^{2} u}{\partial n^{2}}\right|_{\text {wall }},
$$

where $l$ is a viscosity-based mean free path given by $(\mu / p) \sqrt{\pi k T / 2 m}$, as previously described. Other slip models differ slightly in the first-order coefficient, e.g. Ohwada et al. ${ }^{(25)}$ defined it as 1.11 instead of 1.1466 . This difference is well within acceptable bounds. Equation (9) assumes fully-diffuse reflection but the effect of the TMAC can be considered by introducing a factor of $(2-\sigma) / \sigma$ to the first term of this equation. If a Maxwellian scattering model is employed, the fully-developed velocity profile in a channel is given by

$$
u(y)=-\frac{d p}{d x} \frac{H^{2}}{2 \mu}\left[-\left(\frac{y}{H}\right)^{2}+\frac{y}{H}+\frac{(2-\sigma)}{\sigma} 1.1466 K n+2 \times 0.31 K n^{2}\right],
$$


where $d p / d x$ is the pressure gradient in the streamwise direction. A symmetry boundary condition has been employed at the channel centerline in the derivation of Eq. (10). The velocity profile can be nondimensionalized by the mean channel velocity, $\bar{u}$, to give

$$
U(y)=\frac{u(y)}{\bar{u}}=\frac{-\left(\frac{y}{H}\right)^{2}+\frac{y}{H}+\frac{(2-\sigma)}{\sigma} 1.1466 K n+2 \times 0.31 K n^{2}}{\frac{1}{6}+\frac{(2-\sigma)}{\sigma} 1.1466 K n+2 \times 0.31 K n^{2}} .
$$

The results from the LBE approach have been compared with the analytical slip-flow solution of the Navier-Stokes equations, Eq. (11), and show that the effect of the second-order correction for $K n<0.1$ is small. In Fig. 4, the Knudsen number is varied from 0.025 to 0.1 while the TMAC ranges between 0.6 and 0.9 . As previously stated, the second-order correction term is negligible when the Knudsen number is less than 0.1 , so that the symbols representing first- and second-order slip solutions cannot be
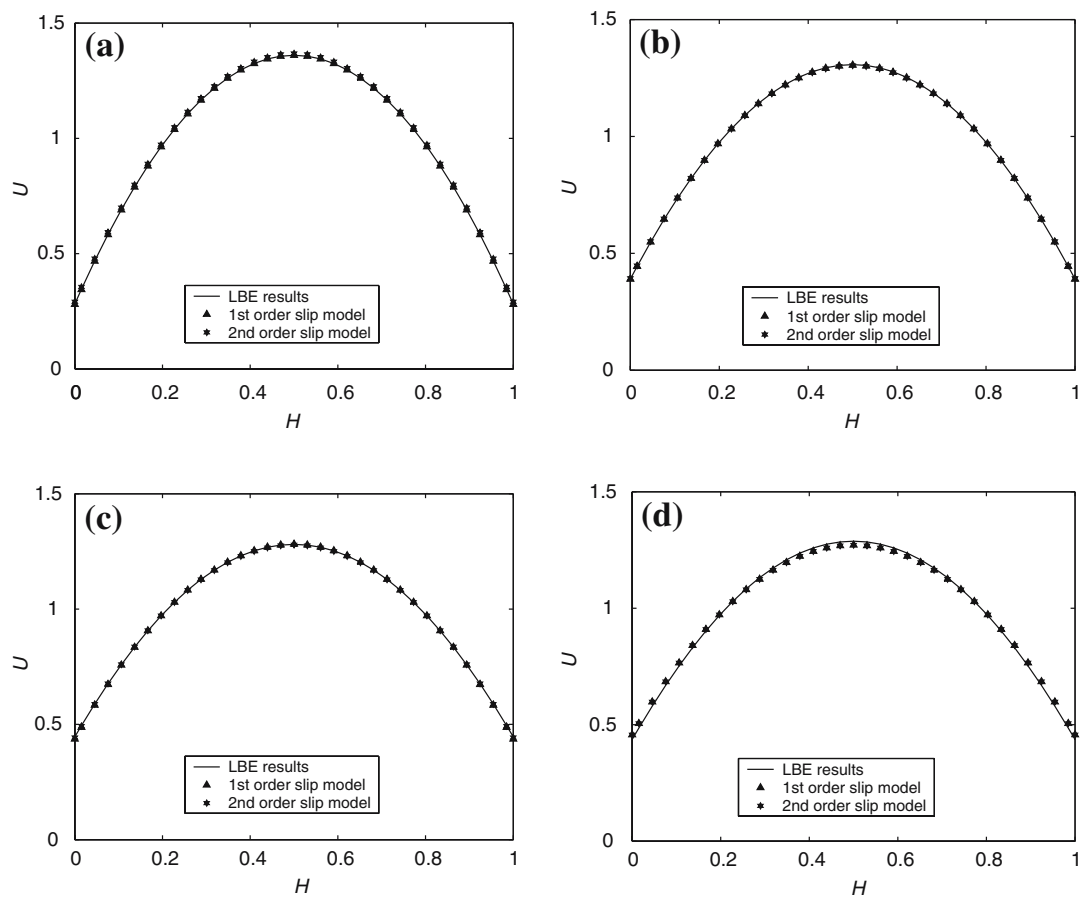

Fig. 4. Non-dimensional velocity profiles for fully-developed channel flows with (a) $K n=$ $0.025, \sigma=0.6$; (b) $K n=0.05, \sigma=0.7$; (c) $K n=0.075, \sigma=0.8$ and (d) $K n=0.1, \sigma=0.9$. 
distinguished. The LBE results are in excellent agreement with the analytical solution for both first- and second-order slip models. When the Knudsen number is increased to 0.1 , where the Navier-Stokes equations are at the limit of their applicability, the difference between the analytical solutions and the $\mathrm{LBE}$ results becomes more noticeable.

In summary, the lattice Boltzmann method offers an alternative approach to the Navier-Stokes equations for modeling low-speed gas flows in the slip-flow regime. In particular, the LBE technique offers the potential to be successfully extended into the transition flow regime due to its particle nature and origins in kinetic theory. The extension of the LBE method into the transition regime, where the Navier-Stokes equations are known to break down, needs further investigation.

\section{ACKNOWLEDGMENTS}

This work was financially supported by the UK Engineering and Physical Sciences Research Council (EPSRC) under grant no. GR/S82978/01. Additional support was provided by EPSRC under the auspices of Collaborative Computational Project 12 (CCP12).

\section{REFERENCES}

1. M. Gad-el-Hak, The fluid mechanics of microdevices - the Freeman scholar lecture, J. Fluids Eng. 121:5-33 (1999).

2. R. Roveda, D. B. Goldstein, and P. L. Varghese, Hybrid Euler/direct simulation Monte Carlo calculation of unsteady slit flow, J. Spacecraft Rockets 37:753-760 (2000).

3. D. B. Hash and H. A. Hassan, Assessment of schemes for coupling Monte Carlo and Navier-Stokes solution methods, J. Thermophys. Heat Transf. 10:242-249 (1996).

4. C. L. Bailey, R. W. Barber, and D. R. Emerson, Is it safe to use Navier-Stokes for gas microflows? in European Congress on Computational Methods in Applied Sciences and Engineering, P. Neittaanmäki, T. Rossi, S. Korotov, E. Oñate, J. Périaux, and D. Knörzer, eds. ECCOMAS 2004 (Jyväskylä, Finland, 2004).

5. X. B. Nie, G. D. Doolen, and S. Chen, Lattice-Boltzmann simulation of fluid flows in MEMS, J. Stat. Phys. 107:279-289 (2002).

6. C. Y. Lim, C. Shu, X. D. Niu, and Y. T. Chew, Application of lattice Boltzmann method to simulate microchannel flows, Phys. Fluids 14:2299-2308 (2002).

7. X. D. Niu, C. Shu, and Y. T. Chew, A lattice Boltzmann BGK model for simulation of micro flows, Europhys. Lett. 67:600-606 (2004).

8. X. He and L.-S. Luo, A priori derivation of the lattice Boltzmann equation, Phys. Rev. E 55:R6333-R6336 (1997).

9. T. Abe, Derivation of the lattice Boltzmann method by means of the discrete ordinate method for the Boltzmann equation, J. Comp. Phys. 131:241-246 (1997).

10. X. Shan and X. He, Discretization of the velocity space in the solution of the Boltzmann equation, Phys. Rev. Lett. 80:65-68 (1998).

11. Y. H. Qian and Y. Zhou, Higher-order dynamics in lattice-based models using the Chapman-Enskog method, Phys. Rev. E 61:2103-2106 (2000). 
12. Y. H. Zhang, R. S. Qin, and D. R. Emerson, Gas surface interaction model in simulation of rarefied gas flows through microchannels using Lattice Boltzmann method, Phys. Rev. E 71:047702 (2005).

13. Y. Qian, D. d'Humières, and P. Lallemand, Lattice BGK models for Navier-Stokes equation, Europhys. Lett. 17:479-484 (1992).

14. X. He and L.-S. Luo, Theory of the lattice Boltzmann method: from the Boltzmann equation to the lattice Boltzmann equation, Phys. Rev. E 56:6811-6817 (1997).

15. F. Sharipov and V. Seleznev, Data on internal rarefied gas flows, J. Phys. Chem. Ref. Data 27:657-706 (1998).

16. S. Chapman and T. G. Cowling, The Mathematical Theory of Non-uniform Gases (Cambridge University Press, 1970).

17. C. Cercignani, Theory and Application of the Boltzmann Equation (Scottish Academic, Edinburgh, 1975).

18. C. Cercignani, R. Illner, and M. Pulvirenti, The Mathematical Theory of Dilute Gases (Springer, New York, 1994).

19. J. C. Maxwell, On stresses in rarefied gases arising from inequalities of temperature, Phil. Trans. R. Soc. 170:231-256 (1879).

20. G. E. Karniadakis and A. Beskok, Micro flows: Fundamentals and Simulation (Springer, New York, 2002).

21. S. Ansumali and I. V. Karlin, Kinetic boundary conditions in the lattice Boltzmann method, Phys. Rev. E 66:026311 (2002).

22. S. Succi, Mesoscopic modelling of slip motion at fluid-solid interfaces with heterogeneous catalysis, Phys. Rev. Lett. 89:064502 (2002).

23. C. Cercignani, Higher Order Slip According to the Linearized Boltzmann Equation, Institute of Engineering Research Report AS-64-19, University of California, Berkeley 1964.

24. N. G. Hadjiconstantinou, Comment on Cercignani's second-order slip coefficient, Phys. Fluids 15:2352-2354 (2003).

25. T. Ohwada, Y. Sone, and K. Aoki, Numerical analysis of the shear and thermal creep flows of a rarefied gas over a plane wall on the basis of the linearized Boltzmann equation for hard-sphere molecules, Phys. Fluids A1:1588-1599 (1989). 